\title{
Heterogeneous field network for power distribution grid based on HPLC and RF adaptive communications technology
}

\author{
Rui Huang ${ }^{1,2,3}$, Yu Xiao ${ }^{2,3}$, Mouhai Liu ${ }^{1,2,3^{*}}$, Zhaoyang Liu ${ }^{2,3}$, Xiaoping Liu ${ }^{2,3}$, Huiting Xu ${ }^{2,3}$, Yunpeng Gao ${ }^{1}$, \\ Maotao Yang ${ }^{2,3}$ and Liuqing Ye $\mathrm{e}^{2,3}$
}

*Correspondence:

279556759@qq.com

${ }^{1}$ College of Electrica

and Information Engineering,

Hunan University,

Changsha 410004, China

Full list of author information

is available at the end of the

article

\begin{abstract}
In order to improve the connection rate and transmission efficiency of field network for power distribution grid, a dual-mode heterogeneous field network with high-speed power line broadband carrier and micro-power radio frequency wireless communication capabilities was designed. First, the topological structure of the field network, the networking process of the central node and the free nodes and the dynamic maintenance mechanism of the network were discussed in detail. Secondly, the routing measurement mechanism for creating a hybrid routing table and the improved layer limit shortest path routing algorithm were presented. On this basis, each node in the network could choose the optimal communication media at any given moment to create communication links with the adaptive data transfer speed according to the real-time hybrid routing table. Finally, the dual-mode heterogeneous field network was applied to the electricity consumption information collection system and tested in the laboratory and jobsite. The test results show that the dual-mode field network was more effective than the single-mode field network in shortening the reading meter time and increasing networking success rate.
\end{abstract}

Keywords: Power distribution grid, Field network, Heterogeneous networks, Highspeed power broadband carrier communication, Micro-power radio frequency wireless communication, Adaptive communications

\section{Introduction}

Field network for power distribution grid is an indispensable part in promoting the construction of electric internet of things [1], which is mainly to solve the communication technology problem of the last mile [2,3]. It can also solve the challenges in the fields of reading intelligent meter, emerging smart grids and interconnecting of smart city facilities in an economic and effective way [4].

Power Line Carrier Communication (PLC) and wireless communication are the two main communication methods in the construction and application of field network for power distribution grid [5]. The power line is generally laid in the wall, and the line is relatively safe and not easy to be damaged. However, due to some factors, such

The Author(s) 2021. This article is licensed under a Creative Commons Attribution 4.0 International License, which permits use, sharing adaptation, distribution and reproduction in any medium or format, as long as you give appropriate credit to the original author(s) and the source, provide a link to the Creative Commons licence, and indicate if changes were made. The images or other third party material in this article are included in the article's Creative Commons licence, unless indicated otherwise in a credit line to the material. If material is not included in the article's Creative Commons licence and your intended use is not permitted by statutory regulation or exceeds the permitted use, you will need to obtain permission directly from the copyright holder. To view a copy of this licence, visit http://creativecommons. org/licenses/by/4.0/. 
as line interference, impedance time-varying, signal attenuation, multipath reflection and so on, the quality of power line carrier communication fluctuates greatly and there is greater distortion in long-distance transmission [6]. Wireless communication has the characteristics of convenience and no wiring. Nevertheless, when encountering obstacles, the signal attenuation is serious, and the communication quality is easily affected by factors such as frequency band, region, climate and environment [7].

In the process of deploying field network for power distribution grid, facing complex environments such as highly urbanization, high-density services, high-rise buildings especially in the underground floor of buildings and so on, the field network for power distribution grid is required to have a higher connectivity rate, transmission rate and reliability, and therefore, it is difficult to meet these requirements of network by using a single communication method [8].

In this study, a dual-mode heterogeneous field network with High Speed Power Line Broadband Carrier (HPLC) and Micro-power Radio Frequency Wireless (RF) communication capabilities was proposed. First, the topological structure of the field network, the networking process of the central node and free nodes and the dynamic maintenance mechanism of the network were discussed in detail. Secondly, the routing measurement mechanism for creating a hybrid routing table and the improved layer limit shortest path routing algorithm were presented. On this basis, each node in the network could choose the optimal communication media at any given moment to create communication links with the adaptive data transfer speed according to the real-time hybrid routing table.

\section{Related work}

Aiming at the shortcomings of power line carrier communication, the power line carrier communication method is improved and the new HPLC communication method based on Orthogonal Frequency Division Multiplexing (OFDM) technology is proposed [9]. A smart meter reading technology based on HPLC is used in customer side universal power internet of things [10]. Aiming at the problem that PLC communication line impedance is difficult to match, an improved L-shaped structure adaptive impedance matching circuit is used to adjust the input impedance of PLC communication [11]. An improved low-voltage power line carrier communication compressive sensing channel estimation method [12] and a low-voltage power line carrier communication active antiinterference technology [13] are used to improve the quality of power line carrier communication. The physical layer security [14], channel characteristics [15] and dynamic channel allocation method [16] of power line carrier communication are studied.

In the construction of field network for power distribution grid, network coverage, communication reliability and deployment economy pose challenges to a single PLC communication method. Some solutions based on PLC using dual-mode communication are proposed, such as PLC + LTE wireless private network mode [17], PLC + RF micro-power wireless mode [18], PLC + WIFI mode [19] and PLC + LoRa wireless communication [20], etc. These solutions mainly adopt the "one main and one auxiliary" hybrid independent networking mode, and each communication mode adopts independent routing after networking. 


\section{Dual-mode heterogeneous field area network}

The dual-mode heterogeneous field network for power distribution grid compromises the merits of HPLC and RF networks and compensates for each other's weaknesses. On the basis of enhancing network coverage, it improves network transmission efficiency and guarantees communication reliability.

\subsection{Topology of dual-mode heterogeneous field network}

The topology of dual-mode heterogeneous field network for power distribution grid is shown in Fig. 1

As shown in Fig. 1, the central node of dual-mode heterogeneous field network is responsible for networking control, network maintenance and management and other functions. It exchanges data with the communication master station of the information management system through a remote communication network, such as optical fiber, GPRS/CDMA, Ethernet and so on, and exchanges data with each node in the field network through HPLC or RF communication. Its corresponding equipment entities are the communication unit of the concentrator. $\mathrm{T}_{1}-\mathrm{T}_{2}$ nodes are proxy node, with three-phase HPLC communication capability and data relay and forwarding function. $\mathrm{A}_{1}-\mathrm{A}_{4}$ nodes have communication capability of A-phase HPLC. $\mathrm{B}_{1}-\mathrm{B}_{5}$ nodes have communication capability of B-phase HPLC. $C_{1}-C_{5}$ nodes have communication capability of C-phase HPLC. All nodes have RF communication ability.

In this network, each node can select the optimal communication medium (HPLC or $\mathrm{RF}$ ) and modulation scheme by using adaptive communication technology at any given time to create a communication link with the best data transfer speed. The selection of this medium and modulation scheme is finished automatically, dynamically and in real time by the equipment itself, without pre-programming or path coding.

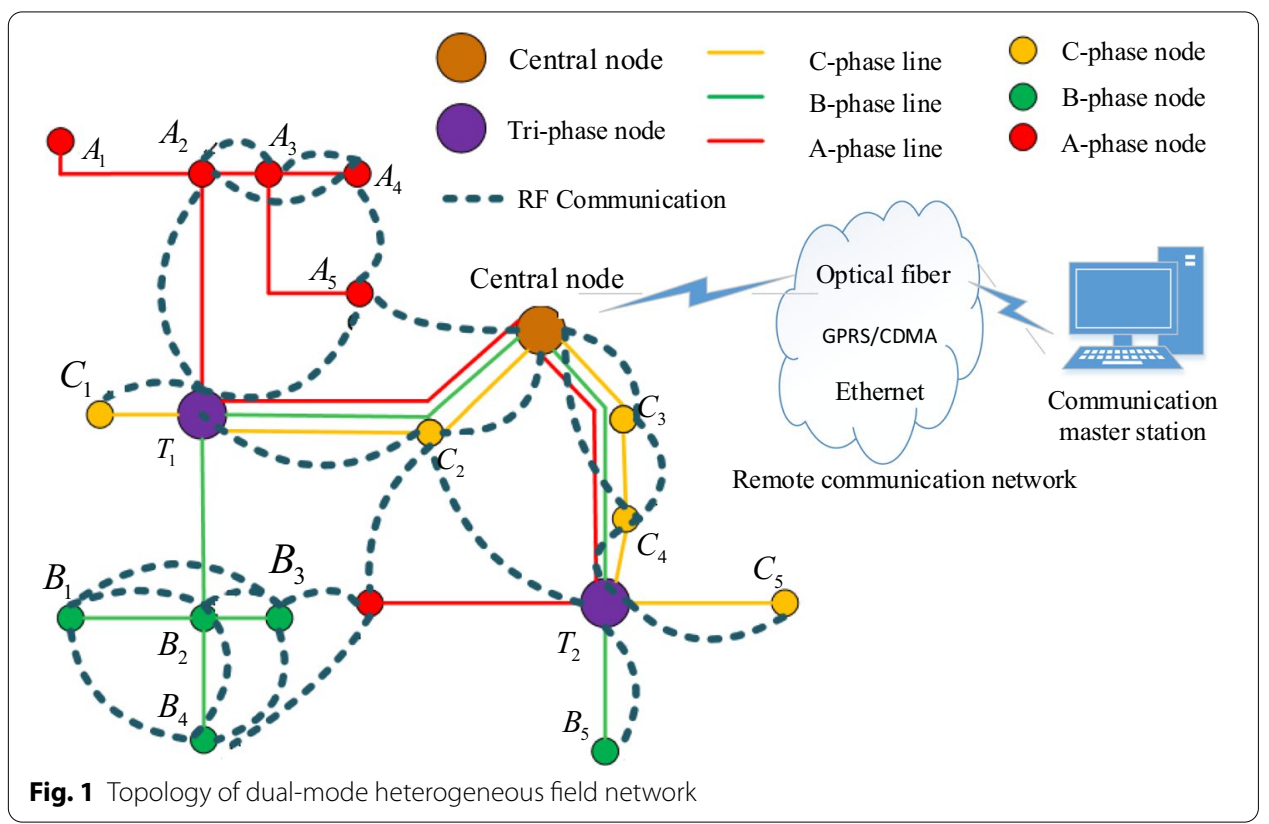




\subsection{Dual-mode communication module hardware composition}

Dual-mode communication module has HPLC and RF communication capabilities, and its hardware composition block diagram is shown in Fig. 2.

As shown in Fig. 2, Microprogram Control Unit (MCU) is the core of the communication module, which mainly runs dual-mode communication protocol and application software. SDRAM and FLASH serve as storage components for programs and data. The HPLC communication unit is mainly composed of four parts: HPLC communication chip, line driver, band pass filter and coupling transformer. The HPLC communication chip selects these chips which support advanced channel coding and multi-carrier OFDM modulation technology. The line driver is used to amplify the transmitted analog signal and the LC band-pass filter is used to filter the received analog signal. The coupling transformer is used to couple the bidirectional analog signal with the power line. The RF communication unit is mainly composed of RF communication chip, impedance matching circuit and signal transmitting antenna. The MCU communicates with the chips, SDRAM and FLASH through the Serial Peripheral Interface (SPI), and communicates with collectors or concentrators supporting the DL/T645 protocol through Universal Asynchronous Receiver-Transmitter (UART) to realize functions such as meter reading and fee controlling.

Some primary communication parameters realized by the dual-mode communication module are shown in Table 1, which fully follow associated enterprise standards of the state grid of China.

\subsection{Dual-mode communication module protocol stack}

The hierarchical design of the dual-mode communication protocol stack aims at easy maintenance, succinct levels, and easy error location. The system does not distinguish between HPLC and RF ports and replaces these ports with a unified and abstract "data channel", making HPLC and RF ports equal and easier unite. The dual-mode communication module adopts a four-layer architecture communication protocol, and the hierarchy of dual-mode communication protocol stack is shown in Fig. 3.

As shown in Fig. 3, The application layer realizes meter reading, node registration, event reporting and other services, and uses the DL/T645 protocol to communicate with

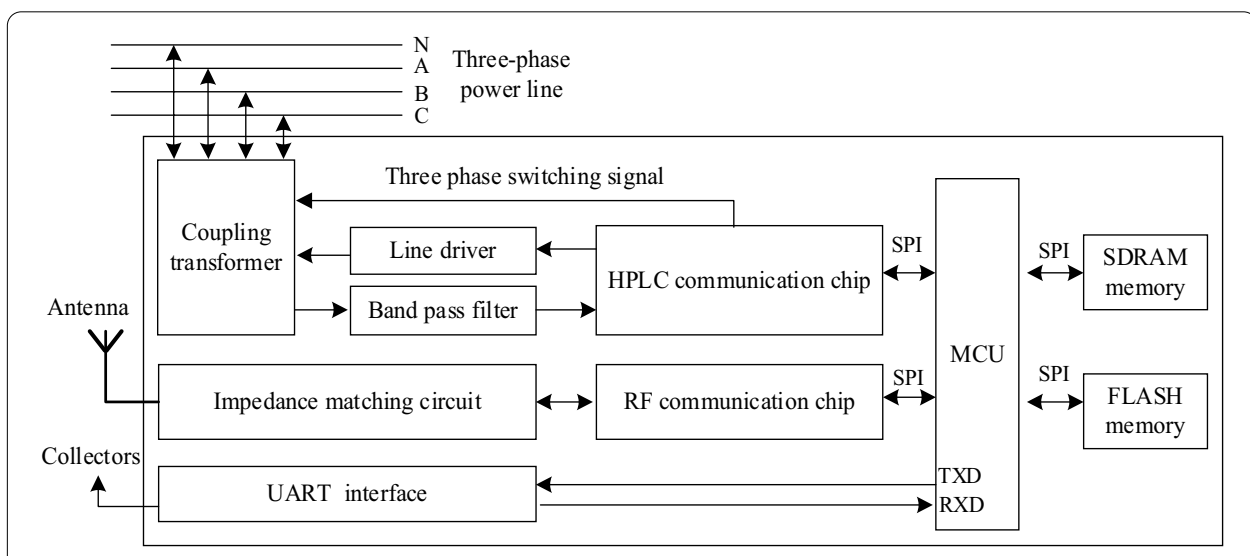

Fig. 2 Hardware composition block diagram of dual-mode communication module 
Table 1 Primary communication parameters of dual-mode communication module

\begin{tabular}{lll}
\hline Mode & Project & Technical indicators \\
\hline HPLC communication & Operating frequency band & $2-12 \mathrm{MHz}$, support segmentation \\
& Power spectrum density & In-band $<-45 \mathrm{dBm} / \mathrm{Hz}$; Out-band $<-75 \mathrm{dBm} / \mathrm{Hz}$ \\
& Modulation mode & Multicarrier OFDM modulation \\
& Transmission rate & Adaptive rate \\
& Anti-attenuation performance & $>85 \mathrm{~dB}$ \\
RF communication & Operating frequency band & $470-510 \mathrm{MHz}$ \\
& Modulation mode & FSK, baseband signal is filtered by GFSK \\
& Modulation frequency deviation & $10 \mathrm{kHz}$ ( 20kbps); $40 \mathrm{kHz}$ (@ 100kps) \\
& Transmission Rate & Support 20kbps and 100kbs \\
\hline
\end{tabular}

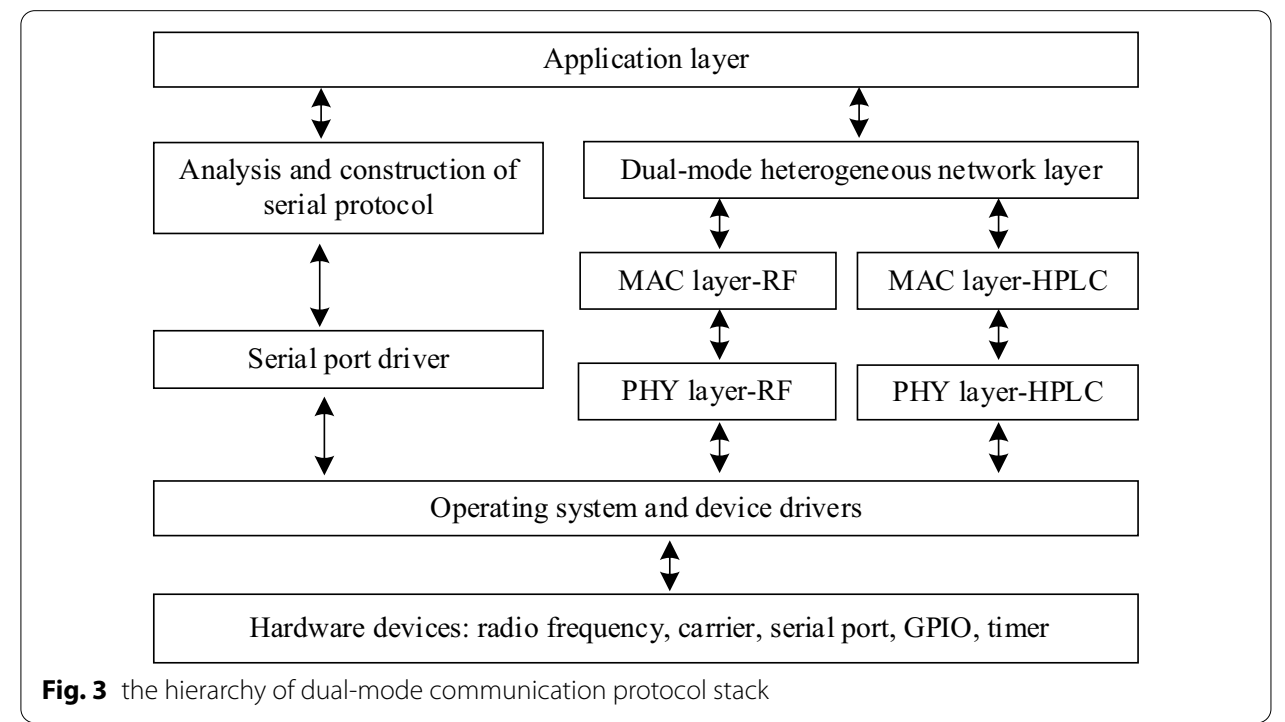

the electric energy meter and collector through the 485 bus. Dual-mode protocol stack includes heterogeneous network layer, HPLC communication link Media Access Control (MAC) layer and Physical (PHY) layer, RF communication link MAC layer and PHY layer. Heterogeneous network layer realizes network networking and maintenance, routing management, and application layer message aggregation and distribution, communicates with the application layer through data transmission and management primitives, and uses adaptive communication algorithm to select RF's MAC layer and HPLC's MAC layer for data exchange. The MAC layer of RF defines these mechanisms of carrier sense multiple access and time division multiple access with collision detection, and uses data and management access points for data exchange with the PHY layer of RF. The PHY layer of RF defines the frequency resources, output power limits, modulation methods, data channel coding methods and channel switching methods required for radio frequency communication. The MAC layer of HPLC uses carrier sense multiple access and time division multiple access to compete for physical channels to achieve reliable transmission of data messages, and uses status and completion primitives for data exchange with the PHY layer of HPLC. The PHY layer of HPLC mainly realizes the modulation 
of the broadband carrier signal and couples it to the power line, receives the broadband carrier signal from the power line, demodulates it into a data message and sends to the MAC sublayer for processing. The protocol stack is based on the management and scheduling to various hardware devices by the operating system.

The message frame encapsulation process of the dual-mode heterogeneous communication protocol is shown in Fig. 4, and the protocol message frame extraction process is just the opposite.

\section{Networking of dual-mode heterogeneous field network}

Only the central node has the right to establish a new network. If the networking process is initiated on any other node, the network layer will terminate the process and notify the upper layer that the request is illegal.

\subsection{Networking by central node}

The networking process of the central node is shown in Fig. 5.

(1) Preparation for networking

In order to expand the coverage of heterogeneous networks, networking preparations are carried out simultaneously on RF and HPLC communication channels. The central node initiates channel scanning at the designated channel group in the MAC layer of its RF to search for possible interference. If a successful energy detection scanning result is received and the energy level of each channel exceeds the acceptable level, then the available channel group is determined. At the same time, the central node initiates the monitoring of the inter-network coordination frame in MAC layer of its HPLC to find out whether there is a neighbor network. If the inter-network coordination frame of the neighbor network is received, it coordinates with the neighbor network and starts the networking after the coordination is successful.

(2) Set network parameters

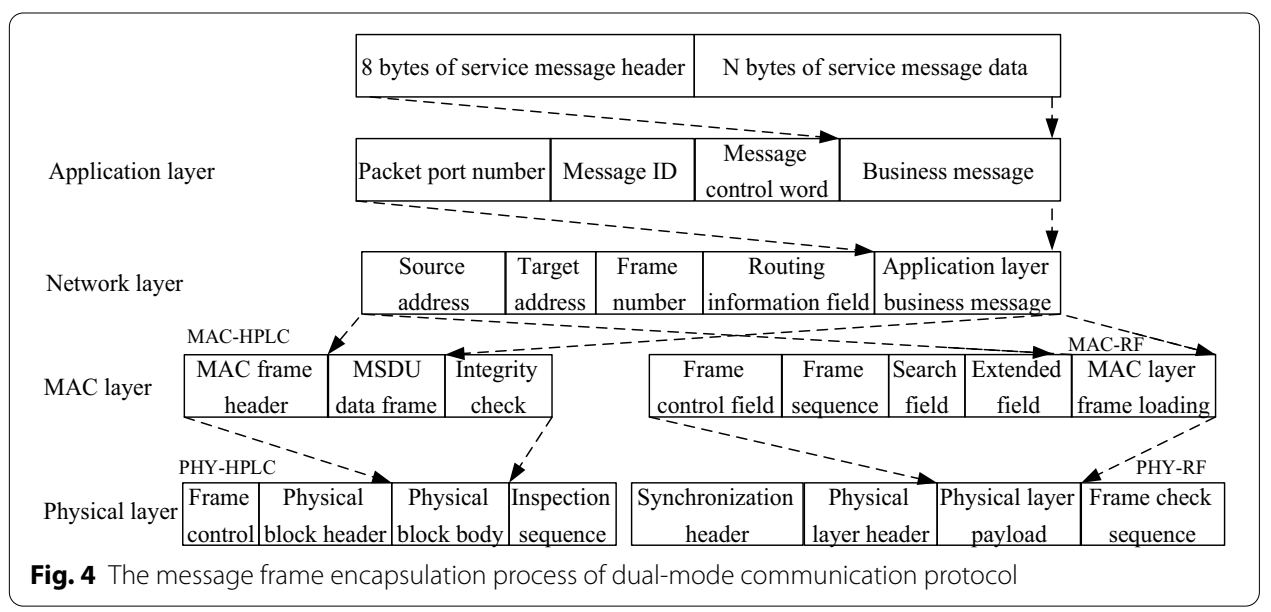




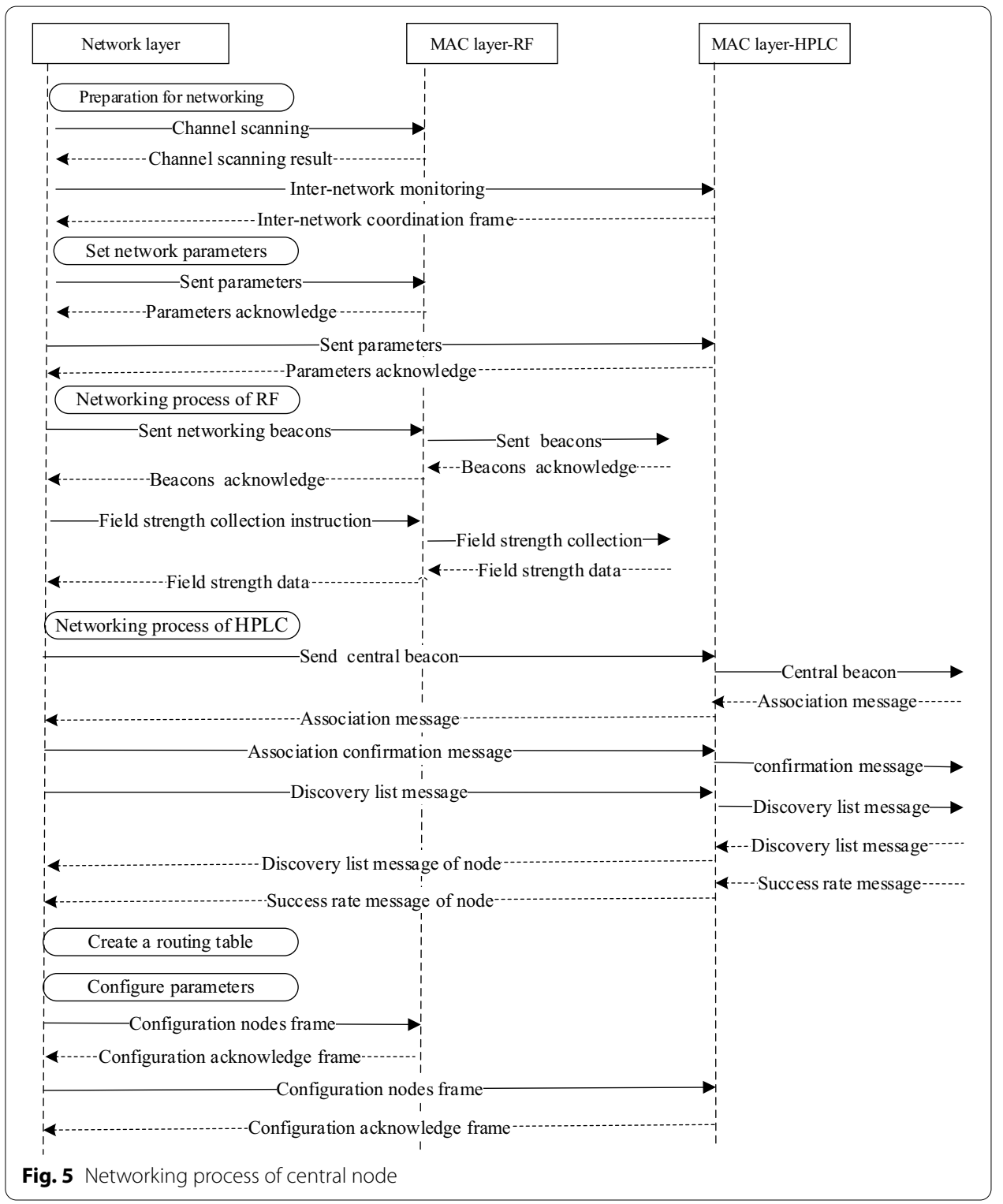

Set the network identity (NID), NID $=$ (central node long address mod 69,069) \&\& 0xFFFF, and fix the Terminal Equipment Identification (TEI) of the central node as 1 . According to the white list stored by the center node, the network scale is determined. The time slot number, level number, beacon round number, maximum beacon forwarding round number and field strength threshold of RF network are set. The time slot length, wireless time slot length, beacon time slot number, CSMA time slot number, Signal to Noise Ratio (SNR) threshold and other parameters of the HPLC network are set.

(3) Initiate the networking process of RF

Networking beacons are sent by the central node in the available channel group of RF and a temporary neighbor table is established according to the replies of each node. 
According to the temporary neighbor table, the field strength collection instruction is sent to collect the field strength of each node.

(4) Initiate the networking process of HPLC

The central node sends the central beacon, arranges the discovery beacon and the proxy beacon transmission through the MAC layer of HPLC, and triggers the layer-by-layer nodes to send the request association message for making an access request. The central node informs the node of its network access result through the association confirmation message. At the same time, the central node broadcasts its own discovery list message regularly and receives the discovery list message and communication success rate report message sent by each node.

(5) Create a routing table

The central node finds the optimal route from the central node to each node in the neighbor information table according to field strength table of the RF link and the request association message, the discovery list message and the communication success rate reporting message of the HPLC link collected by itself form each node, and establishes the RF and HPLC hybrid routing table.

(6) Configure parameters of each node

According to the established routing table, the parameters of each node are configured in sequence. The configuration of the each node parameters is completed by constructing and sending the configuration nodes frame. The path for configuring node parameters is to try all available paths of the node in the routing table from good to bad, until the node's acknowledge frame is received. After the configurations of each node parameters are completed, the networking is finished. The central node compares the networked nodes with the white list to determine whether the network is complete, does not do special processing for incomplete network, and waits for nodes which are not connected to the network to actively join the network.

\subsection{Dynamic network access of free nodes.}

The dynamic network access process of free nodes is shown in Fig. 6 .

The free node first detects network message. If the free node receives network messages from the MAC layer of its HPLC link, it selects the station with good channel quality as its proxy station, initiates an association request message according to the instructions in received message, and then judges whether it is allowed to enter the network according to the received association confirmation message. If yes, the node sets the TEI and sends the discovery beacon. If no, the node sends a network access request frame to each channel group in a polling manner through the MAC layer of the RF link. If a network access acknowledge frame of network access request is received, the node locks the current channel group and records the channel field strength. Then, from all the received network access acknowledge frames, the node selects an optimal path, constructs and sends out a free node ready frame through the MAC layer of its RF link, and configures related parameters according to the received terminal configuration commands from the central node. 


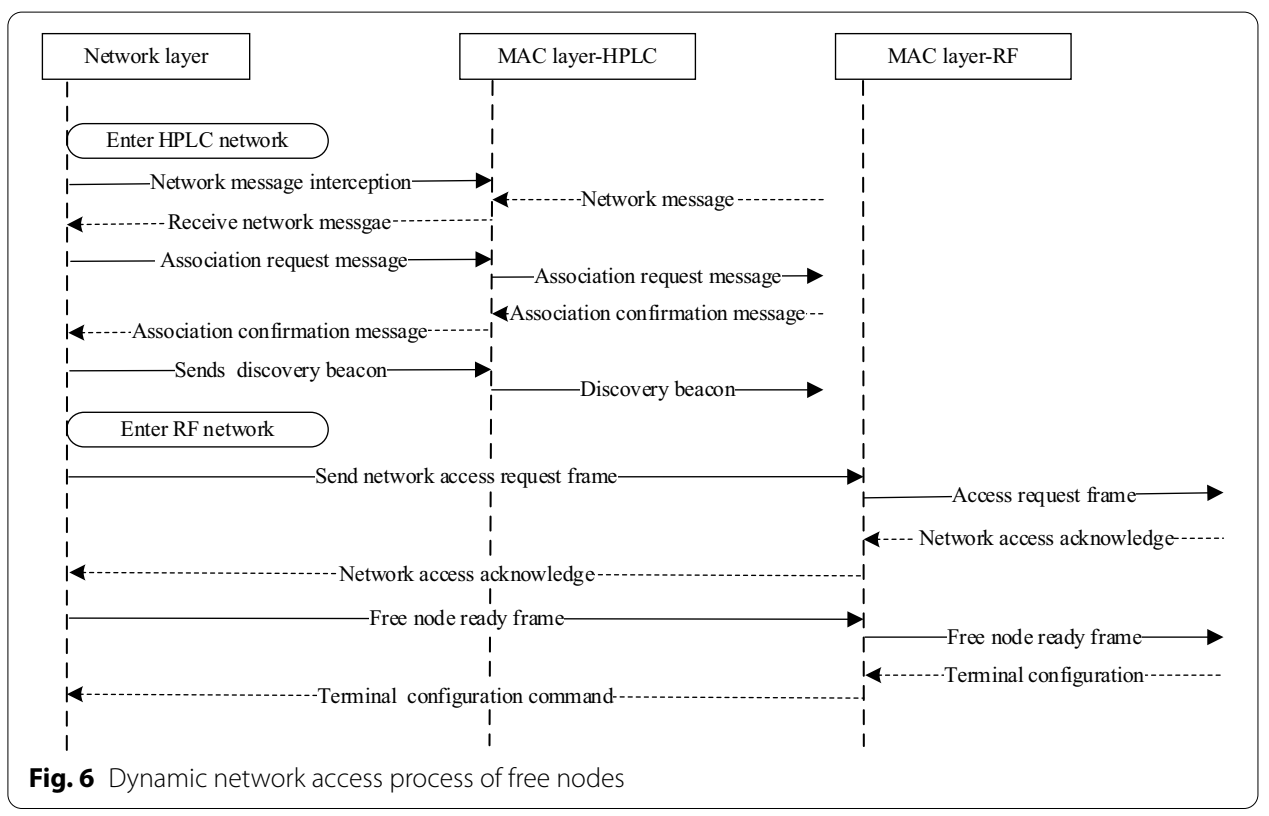

\subsection{Dynamic maintenance of network}

The central node uses the beacon routing parameter notification entry to manage the entire network's beacon cycle, routing cycle, discovery list message sending cycle, and communication success rate reporting cycle.

In a routing cycle, the central node updates the neighbor information of each node in the network and recalculates the optimal route from the central node to each node by receiving the discovery list message, the communication success rate report message and the field strength collection response frame of each node. Then, the central node updates its own routing table and the routing table stored by each node through the configuration parameter frame.

According to the received HPLC link beacons and RF link beacons, each node perceives its neighbor nodes and judges the HPLC channel quality and RF channel field strength of surrounding nodes, generates a discovery list message and performs periodic broadcasts. The node receives the discovery list message which is sent by its neighbor nodes, calculates the communication success rate with neighbor nodes and obtains realtime neighbor node information to form a real-time discovery list. The node evaluates the new proxy site based on the real-time discovery list within a routing cycle, and can initiate an proxy change request to the central node through an proxy change request message.

To prevent routing loops, the routing update time is adjusted by setting suppression timers. When a node receives a route update from a neighbor node which can be accessed before but cannot currently be accessed, it marks the route as inaccessible and starts a suppression timer. If the node receives the updated information from the neighbor node again, which contains a route with a better metric value than the original path, it will mark the neighbor node as accessible and cancel the suppression timer. Before the suppression timer expires, if the metric value contained in the update information received by the node from different neighbors is worse than the previous one, the 
update will be ignored. This method allows more time for updated information to spread throughout the network.

\section{Create hybrid routing table of heterogeneous network}

Each node in the network could choose the optimal communication media at any given moment to create communication links with the adaptive data transfer speed according to the real-time hybrid routing table.

\subsection{Calculation of link metric}

Each node holds the RF network neighbor information table which records the real-time field strength information of the neighbor link and holds the HPLC network neighbor information table which records the real-time uplink and downlink communication success rate and channel quality of links between itself and neighbors.

(1) Evaluate the links

The real-time field strength of the RF link between neighbors is evaluated by the measured value $R_{\mathrm{RF}}$ of the received signal strength indicator (RSSI). When field strength of the RF link is less than or equal to $-120 \mathrm{dBm}$, the value of $R_{\mathrm{RF}}$ is set as 120 . When field strength of the RF link is greater than $-120 \mathrm{dBm}$ and less than $-40 \mathrm{dBm}$, the value of $R_{\mathrm{RF}}$ is set as the absolute value of the field strength value. When field strength of the $R F$ link is greater than or equal to $-40 \mathrm{dBm}$, the value of $R_{R F}$ is set as 40 . When field strength of the RF link is greater than threshold value $-96 \mathrm{dBm}$, the communication cannot be carried out through the RF link.

The communication success rate $S_{\text {HPLC }}$ of HPLC uplink and downlink between neighbors is calculated by multiplying the communication success rate of uplink by the communication success rate of downlink. The channel quality $Q_{\text {HPLC }}$ of the link is evaluated by the signal-to-noise ratio of the original signal. When $S_{\text {HPLC }}$ is lower than threshold of minimum communication success rate or $Q_{\text {HPLC }}$ is lower than threshold value $Q_{\text {gate }}$, communication cannot be carried out through the HPLC link.

\section{(2) Adjust the communication rate of link}

Different message length affects the calculation of communication rate. The communication rate $V_{\mathrm{RF}}$ of RF link and the communication rate $V_{\mathrm{HPLC}}$ of HPLC link are estimated by using the length of most common messages of electricity information meter reading. The communication rate $V_{\mathrm{RF}}$ of RF link is adjusted as $W_{\mathrm{RF}}$ by using field strength $R_{\mathrm{RF}}$ of the RF link. As shown in Eq. (1).

$$
W_{\mathrm{RF}}=V_{\mathrm{RF}} *\left(90-R_{\mathrm{RF}}\right) /(90-40)
$$

In Eq. (1), 90 is minimum threshold of link field strength and 40 is the maximum threshold of link field strength.

The communication rate $V_{\text {HPLC }}$ of HPLC link is adjusted as $W_{\text {HPLC }}$ by using channel quality $Q_{\text {HPLC }}$ and communication success rate $S_{\text {HPLC }}$ of the HPLC link. As shown in Eq. (2). 


$$
W_{\mathrm{HPLC}}=V_{\mathrm{HPLC}} * S_{\mathrm{HPLC}} *\left(Q_{\mathrm{HPLC}}-Q_{\text {gate }}\right) /\left(Q_{\max }-Q_{\text {gate }}\right)
$$

In Eq. (2), $Q_{\text {gate }}$ is minimum threshold and $Q_{\max }$ is the maximum threshold of HPLC link channel quality.

(2) Assignment of link metric

The maximum value of the adjusted communication rate $W_{\text {HPLC }}$ of HPLC link and communication rate $W_{\mathrm{RF}}$ of $\mathrm{RF}$ link is taken as the link metric between the two nodes to ensure that the fastest and most reliable link between the nodes is selected.

\subsection{The creating algorithm of hybrid network routing}

After the nodes in field network for power distribution are deployed, the mobility of nodes is small, nodes exchange data mainly with the central node, and the amount of exchanging data between nodes is small. Therefore, field network for power distribution adopts a centralized routing algorithm, which mainly considers the routing problem between the central node and each node and takes the shortest routing communication time between the central node and the child node as the optimization objective [21].

Considering the time consumed by relay nodes to forward data and the reliability of the network, the number of routing relay nodes is limited. This paper improves the Bellman-Ford algorithm [22], and limits the number of relay nodes. The improved algorithm process is as follows.

The central node builds an undirected graph $G(n, r)$ with n points and $\mathrm{r}$ edges based on the number of nodes in the network and the metric value of the links between nodes. Each point is numbered as $[1 \ldots n]$ separately. The center node is numbered as $1 . w(i, j)$ is the metric value of the link $r(i, j)$ between node $i$ and node $j . e(i)$ is definited as the distance label of central node to node $i, k$ is definited as the number of iterations, and $\mathrm{m}$ is defined as the number of forwarding hops.

1) Initialization. Set $e(1)=0, e(i)=+\infty$, add node 1 to the set $P$. Set the number of iterations as $k=1$, then the distance for the 0th iteration is set as $e_{0}(0)=0, e_{0}(i)=+\infty, i \in[2 \ldots n]$.

2) In the kth iteration, each point $i$ in $P$ is calculated in the order of entry: $e(j)=\min _{(i, j) \in r}\left[e(j), e_{0}(i)+w(i, j)\right]$. If $e(j)<e_{0}(j)$ and $j \in Q$, the points $j$ are added to the set $Q$ and the point $i$ is removed from the set $P$.

3) If the set $Q$ is empty or $k=m$, the algorithm ends. $e(i)$ is the shortest distance from point 1 to point $i$. If the set $Q$ is not empty and $k<m$, execute step (4).

4) Empty the set $P$, transfer all elements in the set $Q$ to the set $P$, and then empty the set $Q$. At the same time, for any point $i \in[1 \ldots n]$, set $e_{0}(i)=e(i)$, and $\mathrm{k}=\mathrm{k}+1$, turn to step (2).

\subsection{Adaptive communication between nodes}

Assuming that the network connection at a certain time is shown in Fig. 7. 


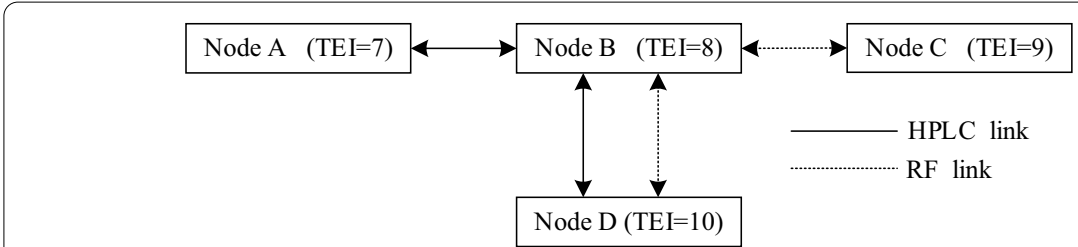

Fig. 7 Diagram of network node connection

Table 2 The hybrid routing table of node B

\begin{tabular}{lll}
\hline Original destination TEl & Forwarding node TEl & Link code \\
\hline 1 & 10 & 1 \\
20 & 9 & 2 \\
5 & 7 & 1 \\
35 & 10 & 1 \\
\hline
\end{tabular}

Assuming that entries in the real-time hybrid routing table of node $B$ are shown in Table 2, where link code 1 represents HPLC communication and link code 2 represents $\mathrm{RF}$ communication.

If node $B(T E I=8)$ needs to report meter reading data to the central node $(T E I=1)$, the node B application layer sends primitives of the MAC layer Service Data Unit (MSDU) to the network layer for transmission. The network layer queries the routing table according to the destination TEI, obtains that the TEI of the forwarding node is 10 and the link code is 1 , and then communicates through HPLC link. According to the destination address $(\mathrm{TEI}=1)$ in the sending primitive, the source address $(\mathrm{TEI}=8)$, MSDU type, the total routing hops and the remaining routing hops, uplink of the broadcast direction, the network layer generates HPLC link MAC frame header, and send data to the HPLC physical layer.

If the node $\mathrm{B}$ receives the MAC message, the MAC message is analyzed. Assuming that the destination TEI of the message is 20 , the TEI $=9$ of the forwarding node and the link code $=2$ are obtained by inquiring the real-time routing table, and the communication will be carried out through RF link. The MSDU of the MAC message is not be corrected, the MAC frame header of the RF link is generated and the data is transmitted through the RF physical layer.

If the destination TEI of the received MAC message is 8 , the destination TEI is the same as the TEI of node B. The message is not be forwarded and is sent directly to the application layer for data analysis and processing.

\section{Experimental results and discussion}

In order to verify the performance of the dual-mode heterogeneous field area network for power distribution grid in the power consumption information system, a test platform with a 6-layer and 16-node planar diamond shaped mesh topology was built in laboratory, as shown in Fig. 8. The communication links of all nodes were adjusted and controlled by the program-controlled attenuator, which could block and open the communication links of these nodes, so as to test the simulation networking and 


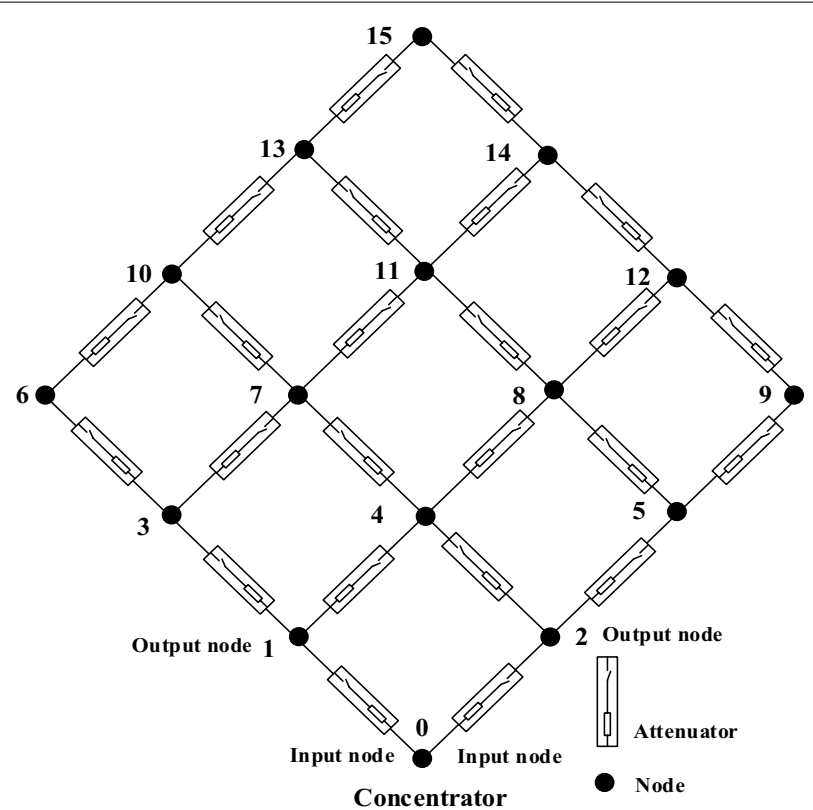

Fig. 8 Topology diagram of platform in laboratory

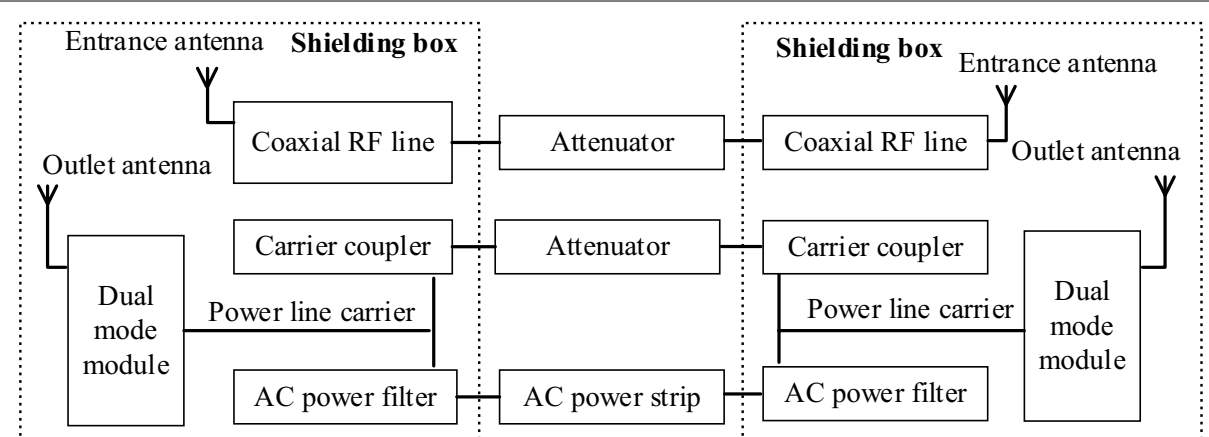

Fig. 9 Connection diagram of test equipment inside and between shielding boxes

communication links switching effect of the dual-mode heterogeneous field area network.

Taking into account the radiation of radio communication, radio links which easily cause radiation were shielded, and each node were placed into shielding box. Dual-mode communication module, HPLC communication module and RF communication module of each node were used for networking test. For the dual-mode communication module test, the connection diagram of the test equipment inside and between the shielding boxes is shown in Fig. 9.

As shown in Fig. 9, after HPLC signal of simulation network enters the shielding box, it is coupled to the power line via the carrier coupler to communicate with the HPLC unit of dual-mode module. The HPLC signal is separated from power supply channel through the AC power filter, so that the power supply channel and the HPLC channel is completely isolated and the power supply channel cannot cause interference to the 


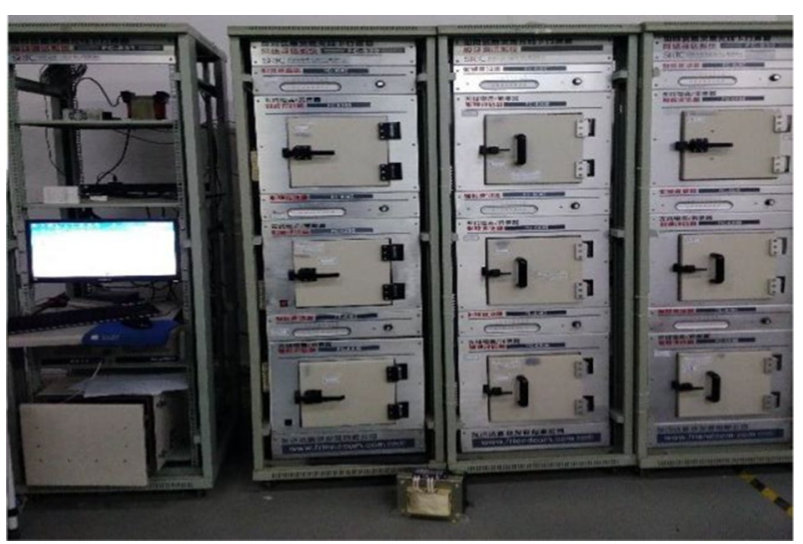

Fig. 10 Test platform of dual-mode heterogeneous field network in laboratory

Table 3 Test data of three kinds of communication mode field network in laboratory

\begin{tabular}{llll}
\hline Performances & Dual-mode (\%) & HPLC (\%) & RF (\%) \\
\hline Networking success rate & 99.68 & 97.96 & 94.34 \\
Daily freezing success rate & 98.64 & 96.13 & 93.61 \\
Power outage and resume reporting success rate & 99.35 & 95.05 & 91.83 \\
Station area recognition rate & 99.85 & 91.75 & 93.45 \\
96-point curve meter reading achievement rate & 99.12 & 97.96 & 95.27 \\
96-point curve meter reading duty cycle & 16.10 & 29.52 & 32.43 \\
\hline
\end{tabular}

HPLC channel. The carrier coupler is connected to the program-controlled attenuator and HPLC signal enters the simulation network. The RF signal of simulation network communicates with the RF unit of dual-mode module through entrance antenna in the shielded box and outlet antenna of dual-mode module, and a small pepper antenna or flat antenna is used in the shielded box to receive and forward the RF signal. The HPLC signal works in the 2-12 MHz frequency band, and the RF signal works in the $470-$ $10 \mathrm{MHz}$ frequency band; therefore, the crosstalk in the shielding box is small. The test platform which was built in laboratory is shown in Fig. 10.

In the test, the Tool-Studio was used as host computer meter reading software, WpsCac-Tool was used as the central node debugging software, and the communication baud rate was set to $9600 \mathrm{bps}$. Some performances of the field network of dual-mode communication, HPLC communication and RF communication, such as networking success rate, daily freezing success rate, and station area recognition rate and so on, were tested by adjusting the attenuation rate of different nodes' attenuator. In continuous testing of 15 days, different network conditions for field network of different communication modes were simulated 100 times. The average test results were shown in Table 3 .

As shown in Table 3, the networking success rate of dual-mode field network has increased by $1.76 \%$ and $5.7 \%$, respectively, compared with that of HPLC field network and RF field area network, the daily freezing success rate of dual-mode field network has increased by $2.6 \%$ and $5.4 \%$, respectively, compared with that of HPLC field network and RF field area network, power outage and resume reporting success rate of 
dual-mode field network has increased by $4.5 \%$ and $8.2 \%$, respectively, compared with that of HPLC field network and RF field area network, station area recognition rate of dual-mode field network has increased by $8.8 \%$ and $6.8 \%$, respectively, compared with that of HPLC field network and RF field area network, 96-point curve meter reading achievement rate of dual-mode field network has increased by $1.2 \%$ and $4.1 \%$, respectively, compared with that of HPLC field network and RF field area network, and 96-point curve meter reading duty cycle of dual-mode field network has reduced by $45 \%$ and $50.4 \%$, respectively, compared with that of HPLC field network and RF field area network. To sum up, the overall performance of dual-mode field network is better than that of HPLC field network and RF field network in laboratory.

The performance of the dual-mode heterogeneous field network for power distribution grid in the power consumption information system was tested in the jobsite. Dual-mode meters, HPLC meters and RF smart meters were installed in No.3, No.4, and No.6 station of Yulong community of Chengdu City in China, where had low, medium and high-rise buildings. The meters were installed in the iron meter boxes on each floor and each meter box had $12 \mathrm{~m}$. The concentrators were installed in the low-voltage power distribution room. The central node's antenna was led out of the power distribution room. Network scale: No.3 station had 157 units, No.4 station had 177 units, and No.6 station had 322 units. The meter reading cycle was 15 min, the number of concurrent reading data items was 3 , and the interval of sending data was $100 \mathrm{~ms}$. Each kind of meter was tested for 20 days, and repetitive networking tests were carried out. The test average data of the periodic business performance of each field network are shown in Table 4. The periodic business performances included Networking Success Rate (NSR), Reading Average Time (RAT), Reading Success Rate (RSR), and Channel Duty Cycle for 15-min (CDC).

As shown in Table 4, the NSR of dual-mode field network could reach 99.66\%, which has increased by $1.0 \%$ and $2.4 \%$ than that of HPLC and RF field network, respectively, RAT of dual-mode field network has significantly shortened by $56.9 \%$ and $72.6 \%$ than that of HPLC and RF field network, respectively, RSR of dual-mode field network could reach $99.28 \%$, which has increased by $2.34 \%$ and $5.30 \%$ than that of HPLC and RF field network, respectively, and CDC of dual-mode field network has depressed by $56.8 \%$ and $62.8 \%$ than that of HPLC and RF field network, respectively. In summary,

Table 4 Test data of three kinds of field network in jobsite

\begin{tabular}{lllllc}
\hline Station & Network type & NSR (\%) & RAT & RSR (\%) & CDC (\%) \\
\hline NO.3 & Dual-mode & 99.65 & 0.27 & 99.12 & 4.66 \\
& HPLC & 98.12 & 0.45 & 97.34 & 7.88 \\
\multirow{4}{*}{ NO.4 } & RF & 95.21 & 0.76 & 95.34 & 8.35 \\
& Dual-mode & 99.78 & 0.24 & 99.31 & 4.79 \\
& HPLC & 98.64 & 0.59 & 96.23 & 11.59 \\
NO.6 & RF & 96.38 & 0.84 & 94.39 & 12.86 \\
& Dual-mode & 99.56 & 0.20 & 99.41 & 7.16 \\
& HPLC & 99.23 & 0.53 & 97.63 & 19.05 \\
& RF & 97.62 & 0.82 & 93.12 & 23.43 \\
\hline
\end{tabular}


the overall performance of dual-mode field network is better than that of HPLC field network and RF field network in the jobsite.

\section{Conclusion}

Field network for power distribution grid is an indispensable part in promoting the construction of electric internet of things, which is mainly to solve the communication technology problem of the last mile. In order to improve the connection rate and transmission efficiency of field network for power distribution grid, a dual-mode heterogeneous field network with high-speed power line broadband carrier and micropower radio frequency wireless communication capabilities was designed in this paper. The dual-mode heterogeneous field network was applied to the electricity consumption information collection system and tested in the laboratory and jobsite. The test results show that the dual-mode heterogeneous field network has the following advantages:

1. The dual-mode heterogeneous field network for power distribution grid compromises the merits of HPLC and RF networks and compensates for each other's weaknesses. Therefore, the connection rate, stability and robustness of the network are improved and the extra maintenance workload of the network is reduced;

2. Each node in the dual-mode heterogeneous field network can choose the optimal communication media at any given moment to create communication links with the adaptive data transfer speed according to the real-time hybrid routing table, hence network hierarchy of the network is reduced and transmission efficiency of network is improved.

3. This dual-mode heterogeneous field network can also be widely used in smart home control systems to realize the reading and control of home appliances such as natural gas meters and water meters.

\section{Abbreviations}

HPLC: High-speed power line communication; RF: Micro-power radio frequency wireless; PLC: Power line communication; OFDM: Orthogonal frequency division multiplexing; MCU: Microprogram control unit; UART: Universal asynchronous receiver-transmitter; MAC: Media access control; TEl: Terminal equipment identification; SNR: Signal-to-noise ratio; MSDU: MAC layer service data unit; NSR: Networking success rate; RAT: Reading average time; RSR: Reading success rate; CDC: Channel duty cycle for 15-min.

\section{Acknowledgements}

We are grateful to the anonymous reviewers who have contributed to the enhancement of the papers completeness with their valuable suggestions.

\section{Authors' contributions}

Rui Huang, Yu Xiao, Mouhai Liu and Zhaoyang Liu contributed to the design and implementation of the research, the analysis of the results, and the writing of the manuscript. Xiaoping Liu, Huiting Xu, Yunpeng Gao, Maotao Yang, and Liuqing Ye conducted the simulations and the experiments. All authors read and approved the final manuscript.

\section{Funding}

This research has been partially supported by Science and Technology Project of State Grid Corporation of China, Ref. 5216A020005K.

Availability of data and materials

The datasets used or analyzed in this study are available from the corresponding author on reasonable request. 


\section{Declarations}

\section{Ethics approval and consent to participate} Not applicable.

\section{Consent for publication}

We agree to the publication of the paper.

\section{Competing interests}

The authors declare that they have no competing interests.

\section{Author details}

${ }^{1}$ College of Electrical and Information Engineering, Hunan University, Changsha 410004, China. ${ }^{2}$ State Grid Hunan Electric Power Limited Company, Changsha 410004, China. ${ }^{3}$ Hunan Province Key Laboratory of Intelligent Electrical Measurement and Application Technology, Changsha 410004, China.

Received: 28 December 2020 Accepted: 9 March 2021

Published online: 19 March 2021

\section{References}

1. T. Yang, F. Zhai, Y.J. Zhao et al., Explanation and prospect of ubiquitous electric power internet of things. Power Syst Autom 43(13), 9-19 (2019). https://doi.org/10.7500/AEPS20190418015

2. T. Chris, S. Simovic, The future of the utility field area network. Nat Gas Electr 35(12), 8-15 (2019). https://doi. org/10.1002/gas.22127

3. J.C. Shi, Z.Y. Xie, Fusion method of low voltage power line and micro power wireless communication for information perception of Power Internet of Things. Electr Power Autom Equip 40(10), 147-157 (2020). https://doi. org/10.16081/j.epae.202009026

4. L.R.M. Castro, N. Ricardo, J.P. Favero et al., The smart grid concept in oil and gas industries by a field trial of data communication in MV power lines. J Microw Optoelectron Electromagn Appl 15(2), 81-92 (2016). https://doi. org/10.1590/2179-10742016v15i2493

5. N. Paul, D. Romina, S. Ioan, Availability Improvements through data slicing in PLC smart grid networks. Sensors 20(24), 236-243 (2020). https://doi.org/10.3390/S20247256

6. C.N. Peng, R.R. Luo, X.D. Wang, Research on a new generation of smart meter-technology supports ubiquitous power internet of things. Electr Meas Instrum 56(15), 137-142 (2019). https://doi.org/10.19753/j.issn 1 001-1390.2019.015.021

7. E. Natalizio, D. Cavalcanti, K. Chowdhury et al., Advances in wireless communication and networking for cooperating autonomous aystems. Ad Hoc Netw 68(1), 13-18 (2018). https://doi.org/10.1016/S1570-8705(17)30196-8

8. K. Yasin, A survey on smart metering and smart grid communication. Renew Sustain Energy Rev 57(8), 302-318 (2016). https://doi.org/10.1016/j.rser.2015.12.114

9. K. Ajit, G.S. Kumar, BER performance analysis of OFDM-based integrated PLC and MIMO-VLC system. IET Optoelectron 14(5), 242-251 (2020). https://doi.org/10.1049/IET-OPT.2019.0132

10. H.X. Wang, R.Y. Tang, Y.D. Wu et al., Research and application of smart meter reading technology based on HPLC in customer side universal power internet of things. Power Syst Protect Control 48(3), 92-98 (2020). https://doi. org/10.19783/j.cnki.pspc.191150

11. N. Jia, W. Song, Y. Wang, Design of adaptive impedance matching network in power-line communication. J N China Electr Power Univ 44(6), 54-60 (2017). https://doi.org/10.3969/j.ISSN.1007-2691.2017.06.09

12. J.S. Zou, D.Y. Pan, Q.W. Zhou et al., An improved compressed sensing channel estimation method for low voltage power line carrier communication. J Chongqing Univ Technol (Nat Sci) 33(11), 155-161 (2019). https://doi. org/10.3969/j.ISSN.1674-8425(z).2019.11.023

13. H. Jing, Z.Y. Zhang, X.H. Pang et al., Active anti-jamming technique for low voltage power line carrier communication. J Nanjing Univ Aeronaut Astronaut 50(5), 701-707 (2018). https://doi.org/10.16356 /j.1005-2615.2018.05.017

14. P.F. Chen, Research on physical layer security of power line carrier communication for cooperative relay. Harbin Inst Technol Harbin China (2020). https://doi.org/10.27061/d.cnki.ghgdu.2020.003088

15. R. Jia, X. Liang, K. Zheng et al., Influence of electrical appliance on the channel characteristics of low voltage power line carrier communication. J Chongqing Univ Posts Telecommun (Nat Sci Edn) 32(3), 411-417 (2020). https://doi.org/10.3979/j.issn.1673-825X.2020.03.010

16. Zou DQ, Dynamic channel allocation method and improvement for power line communication. Harbin Institute of Technology, Harbin of China, 2016

17. C.K. Yang, Y.F. Jian, S.Z. Ren et al., Power LTE network security access technology based on improved authentication protocol. Electr Meas Instrum 56(3), 91-96 (2019). https://doi.org/10.19753/j.issn1001-1390.2019.03.015

18. H.N. Noura, R. Melki, A. Chehab et al., Efficient and robust data availability solution for hybrid PLC/RF systems. Comput Netw (2021). https://doi.org/10.1016/j.comnet.2020.107675

19. B.Z. Zhang, X.C. Chen, D.F. Hu et al., A kind of home electric management system that based on Wi-Fi and ZigBee communication. Microcomput ITS Appl 34(14), 29-32 (2015)

20. X.H. Wang, M. Tang, W.Q. Song et al., Research and application of dedicated LoRa electrical energy acquisition network under state grid standard system. Electr Meas Instrum 57(8), 8-12 (2020). https://doi.org/10.19753 /j.issn1001-1390.2020.08.002 
21. S. Han, L. Zou, A parallel algorithm to answer shortest distance on dynamic graph. Acta Scientiarum Naturalium Universitatis Pekinensis 56(1), 112-122 (2020). https://doi.org/10.13209/j.0479-8023.2019.113

22. AbuSalim SWG, Ibrahim R, Saringat MZ et al. Comparative analysis between Dijkstra and Bellman-Ford algorithms in ahortest path optimization. In: International conference on technology, engineering and sciences (ICTES), Penang, Malaysia, 2020; vol 917, pp12077-12086. https://doi.org/https://doi.org/10.1088/1757-899X/917/1/012077

\section{Publisher's Note}

Springer Nature remains neutral with regard to jurisdictional claims in published maps and institutional affiliations.

Submit your manuscript to a SpringerOpen ${ }^{\circ}$ journal and benefit from:

Convenient online submission

- Rigorous peer review

- Open access: articles freely available online

High visibility within the field

Retaining the copyright to your article

Submit your next manuscript at $\boldsymbol{\Delta}$ springeropen.com 